\title{
A Users' Satisfaction Driven Scheduling Strategy for Wireless Multimedia QoS
}

\author{
Leonardo Badia, Michele Boaretto, Michele Zorzi * \\ \{lbadia, mboaretto, zorzi\}@ing.unife.it, \\ DipInge, University of Ferrara, Italy
}

\begin{abstract}
In this work, we exploit game-theoretical concepts to depict the behaviour of multimedia users for the Radio Resource Management. Moreover, we also include pragmatic economic considerations, which allow studies of provider's revenue and possible charging mechanisms. These concepts are in particular applied to HSDPA scheduling procedures, whose main aim is to improve the performance of $3 \mathrm{G}$ networks and to allow extensions to a plethora of services. We briefly discuss a model for the users' satisfaction that includes both perceived QoS and pricing, already proposed to determine the QoS provisioning and network dimensioning. We apply the users' satisfaction function of this model in the scheduler. In this way we achieve improvements of the QoS as it is seen from the users' point-of-view, i.e., by involving the satisfaction of service constraints but also paid price. This analysis will be extended also to the provider's side, with considerations on the achievable revenue, that is an important aspect to take into account in service supplying.
\end{abstract}

\section{Introduction}

The recent standardisation of High Speed Downlink Packet Access (HSDPA) interface opens up the availability of a faster adaptation to the channel state for packet transmission in WCDMA networks. In HSDPA, the downlink channel is shared among all users, basically in a TDMA-like fashion. Thus, the following factors have an impact on the performance and determine different outcomes of the offered Quality of Service (QoS): the radio propagation conditions, the scheduling technique and the coexistence of many users in the same sector.

In [1] the concept of Channel-State Dependent Scheduler is introduced, i.e., it is shown that it is fundamental for the scheduling technique to be aware of the user's instantaneous link state condition. A general conclusion, that is still valid in the case under exam, is that the overall throughput for the sector can be maximised if the scheduler uses its knowledge of the channel state, so that only users with good channel are served. On the other hand, to meet QoS constraints, it can be necessary to supply a certain degree of fairness, that implies to serve also users with bad channel condition.

The issue of the satisfaction of users' QoS requirements is not trivial, as it can be related to the economic problem for the provider to achieve an adequate revenue. For a real operator, this aspect can not be neglected, since the network maintainance is possible only if the costs of service provisioning are overcome.

\footnotetext{
* This work has been partially supported by Ericsson Research. Proceedings of QoFIS 2003, Stockholm (Sweden). Published by Springer Verlag in Lecture Notes on Computer Science. (C) Springer Verlag 2003.
} 
In order to emphasise the analysis of this matter in more depth, we adopt a utilitybased approach. Utility functions have been widely used in the recent technical literature, with particular focus on the modeling of users' satisfaction in Radio Resource Management (RRM) problems [2] [3]. Moreover, for the scheduling, several solutions can be seen as application of a utility-based framework: the scheduling issue is often seen and solved as a problem of priorising users in a queue, and this can be done by defining appropriate weights, like in the Weighted Fair Queuing (WFQ) [4] or $\mathrm{WF}^{2} \mathrm{Q}$ [5] schedulers. In [6] a mathematical formulation of the RRM issue, directly applicable to our case, has been developed, by involving utility functions with assigned properties, that depict the soft tunability of the QoS requirements. Hence, the RRM goal is seen as a strategy of maximizing the system utility, that in this approach includes only the users' welfare.

In [7] this concept was extended, by including also pricing and demand effects. This means that the welfare of the network is also related to the money exchange between user and service provider. In fact, users are likely to be satisfied if they obtain an acceptable QoS, but also if they pay a fair price for the offered service. Thus, the users' satisfaction should be an increasing function of the offered QoS; however, the higher the price, the lower the satisfaction. Hence, we should refer to economics in two directions, i.e., for utility functions and for what concerns the pricing strategies [8].

In this work, we take into account this trade-off between users' and provider welfare by considering a model of the users' satisfaction as previously discussed. Moreover, we apply different strategies of scheduling on the HSDPA channel within the satisfaction framework. It has also to be taken into account in the welfare maximisation, that the operator can not offer the service without adequate revenue. In this way, the provider welfare can be expressed in economic terms; we will discuss how a satisfactory revenue can be obtained from the given algorithms, and we compare different scheduling techniques also in terms of achievable revenue. Thus, the main contribution of the present paper is to investigate if well-known strategies which obtain an optimal scheduling under the technical point-of-view, i.e., by maximising the throughput, are efficient if revenue is taken into account, or there is margin of improvement, and how large.

The work is organised as follows: in Section 2 we present the analytical model for the users' satisfaction, including both pricing and utilities. In Section 3 we discuss different scheduling strategies under the theoretical point-of-view and we outline how they can be modified to take into account the revenue improvement aspect. Section 4 presents simulation results and Section 5 concludes the work.

\section{Model for the Users' Satisfaction}

We present a model that employs the concept of utility function, used in microeconomics to classify and sort the customer preferences. Thus, we are considering the distribution among the users of a scarce resource, represented with a generic qualityrelated parameter $g(g \geq 0)$, and the results of this operation are seen with a mapping through a utility function $u(g)$. In our study, the utility and the preferences are related to multimedia wireless services, in particular under the point-of-view of the Radio Resource assignment. Hence, $g$ represent the assigned network resource. Note that the analysis can be easily extended by replacing $g$ with a multi-dimensional vector. For the scheduling issue, $g$ can be identified with the assigned rate. In this analysis we will study the rate assignment $a$ priori, i.e., as it is before the transmission. Thus, $g$ is the assigned transmission rate on the HSDPA channel. Indeed, to have a better adherent model we should replace this parameter with the throughput in terms of correctly delivered packets, as this is a more appropriate metric to depict the customer satisfaction. 
However, it is still possible to do this in the given framework, with only small modifications. In fact, note that these two quantities (assigned rate and achieved throughput) are strictly connected.

If there are $N$ users in the network, in general each user will have a different utility function $u_{i}(g)$, with $i=1,2, \ldots, N$. The utility level of the $i$ th user will depend on the assignment of $g_{i}$. Even though we do not investigate in detail how the utility functions can be derived in different networks, we exploit some of their general properties. For example, since a larger amount of bandwidth can be useless but can not hurt, the utility functions $u(g)$ are assumed to be non decreasing functions of $g$. On the other hand, the economic law of diminishing marginal utilities states that the improvement of the quality due to a larger amount of $g$ becomes smaller as $g$ increases. Formally:

$$
\forall i=1,2, \ldots, N \quad \frac{\mathrm{d} u_{i}(g)}{\mathrm{d} g} \geq 0 \text { and } \lim _{g \rightarrow \infty} \frac{\mathrm{d} u_{i}(g)}{\mathrm{d} g}=0 .
$$

The right part of previous equation can be also almost equivalently expressed as the observation that there is a value $g_{\max }$ such that the utility $u\left(g_{\max }\right)$ is the upper limit for each $u_{i}(g)$, in other words:

$$
\forall i=1,2, \ldots, N \lim _{g \rightarrow \infty} u_{i}(g) \approx u_{i}\left(g_{\max }\right) .
$$

This formulation is not fully equivalent to (1) but it is almost always verified when technological constraints are involved. In this case, $g_{\max }$ can be seen as the maximum amount of resource that can be received from the technological support (user terminal). Note that the conditions on the utilities expressed above imply that every $u_{i}(g)$ is necessarily concave for $g$ greater than a given value. In this work we will consider also 0 as the minimum achievable utility, that corresponds to the utility of not receiving the service at all, i.e. $u(0)=0$. This condition can also be changed if run-time service degradation are considered, as in this case the utility could go even below 0 ; in fact, it is commonly assumed preferable to be not admitted at all than to be disconnected from the network while receiving the service. Thus, a lowest utility equal to 0 corresponds to a condition of ideal Admission Control.

Different types of utility functions can be introduced to model different kinds of service, as discussed in [9]. For example, discrete-value utilities (i.e., combination of step functions) are an appropriate characterisation for the simplest kinds of traffic, like GSM-like voice call. Here, the aim of introducing the utilities is only to determine whether the service is acceptable or not, but it does not matter how much it is appreciated.

According to the chosen mathematical representation, there are several ways to combine the users' utilities. Commonly, the utilities are assumed to be additive [3], thus, their aggregate is simply a sum; in other models, different combinations are proposed. In any case, however, the aggregate of the users' utilities contributes to the network welfare, and the first goal of the RRM can be seen with a naïve point-of-view as the welfare maximisation.

This leads to a straightforward application of the general model as presented in [6], by considering the RRM as an optimisation problem:

$$
\begin{gathered}
\max W(\mathbf{g}) \quad W(\mathbf{g})=\sum_{i=1}^{N} u_{i}\left(g_{i}\right) \\
\text { s.t. } \sum_{i=1}^{N} g_{i}<K_{\text {max }},
\end{gathered}
$$


where $W$ is the network welfare, defined as an aggregate of the utilities and thus function of the vector $\mathbf{g}$ of the assigned $g$ 's. Equation (3) can be replaced with other similar aggregation of the utilities. Also the kind of capacity characterising the network can be represented with different conditions than Eq. (4). In this case, Equation (4) represents a hard capacity system, that is a TDMA- or FDMA-like systems with fixed assigned maximum quantity $K_{\max }$ of allocable resource. In general it can be written: $\mathcal{K}(\mathbf{g})<K_{\max }$ with an appropriately defined capacity constraint $\mathcal{K}$. The main point, yet, is that in the expression of the welfare $W(\mathbf{g})$ only the single user utilities are worth.

It seems to be more realistic to consider at the same time instead also the effect of the pricing [10] This is not only suggested for the sake of a realistic model. In fact, the strategies of charging users for the offered service generate revenue, by improving at the same time the network management. For example, a cheap service is also likely to be abused of. Moreover, between the pricing strategies that are almost equally acceptable by a willing-to-pay user, the operator should choose the one that provides the highest revenue, since in this way its own satisfaction is increased. As long as this can be done without decreasing too much the users' welfare, this implies indeed a more efficient resource usage; in other words, wastes are avoided.

However, it should also be considered that in general a price variation can greatly affect the users' demand, resulting in a translation of (3)-(4) into different optimisation problems. From the point-of-view of the network provider, it can be assumed that users that do not face both adequate QoS and affordable price are unsatisfied customers. Thus, it can be assumed that these users pay only a fraction of the tariff they are supposed to. This can be seen also with a probabilistic approach: if we map the satisfaction $A_{i}$ of the $i$ th user into the range $[0,1]$, we can express the expected value of the revenue as

$$
R=\sum_{i=1}^{N} A_{i} p_{i}
$$

where $p_{i}$ is the price paid from the $i$ th user. This model was proposed in [7] to depict the users' satisfaction coming from a static heuristic rate assignment. Actually it is possible to extend the framework to the case under exam, i.e., a more detailed scan of the solution to the allocation problem, which can constitute an adequate scheduling strategy.

In more detail, $A_{i}$ represents the satisfaction, or the acceptance probability of the $i$ th user. The latter denomination better identifies the meaning of this parameter, that is indeed probabilistic. In sufficiently large networks, or after a sufficiently long run, it is likely that $A_{i}$ is also the fraction of satisfied customers, i.e., the ones who keep paying for the service without abandoning it or being driven to other operators.

There are several possibilities to define $A_{i}$, with different kinds of parameters. However, the basic dependences are the offered QoS, that is better described through its perception represented by the utility $u_{i}$, and the paid price $p_{i}$. Hence, we will consider $A_{i}=A\left(u_{i}, p_{i}\right)$ for each $i$, by assuming that every user in the network follows the same decision criterion to decide whether they are satisfied or not. On the other hand, note that the users are differentiated in the perception of the offered service $\left(u_{i}(g)\right.$ changes according to the index $i$ ). Also the price can vary among the users.

For each definition of the acceptance probability, the following properties have to hold:

$$
\begin{aligned}
\forall(u, p) \neq(0,0): \quad & \frac{\partial A}{\partial u} \geq 0, \lim _{u \rightarrow 0} A(u, p)=0, \lim _{u \rightarrow \infty} A(u, p)=1, \\
\frac{\partial A}{\partial p} & \leq 0, \lim _{p \rightarrow 0} A(u, p)=1, \lim _{p \rightarrow \infty} A(u, p)=0 .
\end{aligned}
$$


where the limits $u \rightarrow \infty, p \rightarrow \infty$ should be intended only in a mathematical sense, as it is likely that they do not represent a feasible situation. For example, as discussed above, $u$ is upper-bounded to $u\left(g_{\max }\right)$. This justifies the fact that the condition $u_{i}=p_{i}=\infty$ could not have a completely coherent definition with the above properties. Note that even the value of $A$ on the edge point $u_{i}=p_{i}=0$ is also difficult to be properly defined; however, this point does not affect the definition as $A_{i} p_{i}$ of the revenue contribution. In fact, this case represent a user not admitted, thus the paid tariff is 0 , and this is verified for any value we decide to assign to $A(0,0)$ in the interval $[0,1]$.

A satisfactory definition of $A$ is henceforth:

$$
A\left(u\left(g_{i}\right), p\left(g_{i}\right)\right) \triangleq 1-\exp \left(-C \frac{u^{\mu}}{p^{\epsilon}}\right) .
$$

Note, however, that almost identical results can be obtained from any expression of $A(u, p)$ that verifies the above properties. Furthermore, note that in the more general case of $p$ seen as a function of $g$ it is realistic to require that also $p(g)$ is an increasing function of $g$.

Thus, the total revenue expressed by Equation (5) can be rewritten as $R(\mathbf{g})$, where $\mathbf{g}=\left(g_{1}, g_{2}, \ldots, g_{N}\right)$ is the vector of the assigned $g$ 's. With these considerations, the optimisation problem (3)-(4) can be rewritten as

$$
\begin{aligned}
\max R(\mathbf{g}) & =\sum_{i=1}^{N} A_{i}\left(g_{i}\right) p_{i}\left(g_{i}\right) \\
\text { s.t. } \mathcal{K}(\mathbf{g}) & <K_{\text {max }} .
\end{aligned}
$$

In this work we do not consider analytical solutions to the problem. Instead, we analyse the behaviour of classical scheduling algorithms for what concerns the revenue and present possibilities of improvement. Moreover, we will quantify how much the revenue can be increased with appropriate techniques.

\section{Scheduling Algorithms Framework}

Let us present how classical scheduling algorithms can be extended to the HSDPA release of UMTS. The choice of the scheduling strategy has a major impact on the system performance: however, in HSDPA the channel conditions might be fastly tracked to improve the system throughput. In this kind of system the MAC features are located in the node- $\mathrm{B}$, in order to evaluate the rapid variations of the wireless channel, i.e., fast fading.

In a wireless network, considering the instantaneous radio conditions is a fundamental task, because of the location-dependent and bursty errors typical of this kind of systems. For example, a user in a fading dip may experience a bad channel and may be unable to transmit for a certain period of time. The scheduling framework has to consider the channel conditions and to give priority to users that perceive a clean channel; users with a poor SIR will be delayed until they have a better propagation scenario.

Such a policy permits the maximisation of total throughput because it minimises packet retransmissions. Nevertheless some degree of fairness is required, in order to prevent users' starvation. In a Round Robin (RR) scheduling resources are allocated to the communication link without taking into account the channel conditions but only on a sequential basis, with a high degree of fairness but with the potential risk of not considering the propagation scenario, causing a possible high number of retransmissions. Due to the poor performance exhibited in this sense by the pure RR scheduler, in the 
following we will not analyse this strategy. In fact, to guarantee the QoS requirements it is necessary to find a trade-off between a pure SIR-based heuristic and a round robin scheduling, i.e., between the throughput maximisation and the number of users that can achieve a given QoS.

We consider various kinds of heuristics involved in the scheduling process. For example, we might introduce a traditional SIR-based heuristic, called C/I, with a greedy assignment of the available resources [11]; such a policy permits to obtain the maximum sector throughput, but users that perceive a bad channel may have a poor assignment of resources. In other words, the sharing of the codes is basically characterised by a high degree of unfairness.

As opposed to such a policy we introduce, as an original contribution, a utilityfunction-based assignment, with the aim of increasing the achievable revenue. Considering an assigned rate dependent on a utility function permit not only to obtain a better degree of fairness, but also a generally better allocation, in particular in terms of fairness. This happens because the rate is assigned by following the perceived user-utility and not only the channel state.

The policies will be compared in terms of revenue and users' admission in order to highlight the consequences on the provider side. In a utility-based approach it is possible to assign the resources according to more complex issues compared to a simple C/I policy; user parameters like SIR, buffer state, deadline of the packets can be considered and mixed in a more efficient manner.

In our proposed strategy the scheduling process starts from a solution obtained with a greedy heuristic and modifies this assignment giving more resources to the user with the highest marginal utility, in order to improve the total sector utility. Resources are subtracted to user with the minimum marginal utility, to obtain a variation of the total utility as little as possible. This algorithm is based on a local search of the optimal solution, ending when the goal function reaches a local maximum.

The heuristic for the starting solution affects the result of the local-search in two ways: firstly, it should be a good solution in itself, as this improves the convergence of the algorithm. Secondly, it has to be general enough to scan the solution range without being specialised to a peculiar subset of cases. Even though the solution coming from the $\mathrm{C} / \mathrm{I}$ algorithm is good, we prefer for these reasons to choose another option.

In the following, the starting solution $g_{i 0}$ for the rate assignment for the $i$ th user will be determined by means of the marginal utility, i.e., $u_{i}^{\prime}(g)$. In particular, $g_{i 0}$ is the highest feasible value that implies a marginal utility equal to a given threshold $\vartheta$. According to the value of $\vartheta$, different heuristics are determined in this way. The meaning of this heuristic, and the meaning of $\vartheta$, are the following: the marginal utility $u^{\prime}(g)$ represents the increase of the QoS perception for increasing $g$. Thus, $g_{i 0}$ is the highest value that can guarantee a relative increase larger than $\vartheta$; beyond this value, the increase is always lower. Obviously the choice of $\vartheta$ depends on the kind of strategy that the provider wants to follow in the assignment, as a value of $\vartheta$ close to 0 implies to assign almost the maximum meaningful value, that is, $u_{i}\left(g_{i 0}\right) \approx u_{i}\left(g_{\max }\right)$. On the other hand, the larger $\vartheta$, the lower the starting assigned rate $g_{i 0}$.

After this initial condition, the assignment can be modified, by means of a localsearch algorithm obtaining a local optimum solution.

Moreover, the revenue will also depend on the pricing strategy. Thus, the choice of the function $p(g)$ should be indicated to clarify the above definition of revenue, as given in Equation (5). In the literature [12], different pricing strategies have been proposed, and obviously the pricing strategy choice heavily affects the value of the total revenue. In this work we will consider two kinds of pricing policies, mainly for their simplicity 
of concept. The first one is a flat price strategy, i.e., the price is fixed for any value of the assigned rate. The second policy represents instead a simple usage-based pricing with linear price. This means that $p(g)=k g$ is linearly related to $g$ through a given constant $k$. It is interesting to observe that in Equation (5) there is expressed a double dependence of the revenue on the pricing, as also $A_{i}$ is a function of the price.

For what concerns the utilities $u_{i}(g)$, they are assumed to be sigmoid functions of $g$, with general definition:

$$
\forall i=1,2, \ldots, N \quad u_{i}(g) \triangleq \frac{\left(g / K_{i}\right)^{\zeta_{i}}}{1+\left(g / K_{i}\right)^{\zeta_{i}}},
$$

where the parameters $K_{i}>0$ and $\zeta_{i} \geq 2$ depend on the index $i$, so that different users may follow different utility functions. In the simulations, $K_{i}$ and $\zeta_{i}$ are randomly generated with uniform distribution within a given interval.

Table 1. List of Parameters of Simulation Scenario

\begin{tabular}{c|c}
\hline Parameter (symbol) & value \\
\hline cell radius $(d)$ & $250 \mathrm{~m}$ \\
gain at $1 \mathrm{~m}(A)$ & $-30 \mathrm{~dB}$ \\
Hata path loss exponent $(\alpha)$ & 3.5 \\
shadowing parameter $(\sigma)$ & $4 \mathrm{~dB}$ \\
Doppler frequency $\left(f_{d}\right)$ & $2 \mathrm{~Hz}$ \\
mean SNR at cell border & $40 \mathrm{~dB}$ \\
max assignable rate $\left(g_{\max }\right)$ & 96 codewords \\
\hline utility parameter $(\zeta)$ & $5.0 \div 8.0$ \\
utility parameter $(K)$ & $0.2 \div 6.0$ \\
acceptance prob. parameter $(C)$ & 0.5 \\
acceptance prob. parameter $(\mu)$ & 2.0 \\
acceptance prob. parameter $(\epsilon)$ & 4.0 \\
\hline
\end{tabular}

With these definitions, in the next we will study the behaviour of the classical C/I scheduling policy against our proposal introduced to improve the revenue by simulations. However, this aspect of the scheduling could be studied even from the point-ofview of finding a theoretically optimal resource allocation.

\section{Results}

In this Section we will present the results relative to the HSDPA interface obtained with a UMTS simulator developed at the University of Ferrara, in which detailed user dynamics have been implemented. The simulation environment consists of a $3 \times 3$ hexagonal cells' structure. The cells' cluster is wrapped onto itself in order to avoid the "border effect". In radio channel propagation, path loss, fast fading and shadowing have been included. By considering the environment mobility, a non-zero Doppler frequency is assigned, even though stationary users are considered. Table 1 reports the data for the simulation scenario and the Acceptance-probability model.

The compared scheduling strategies are the previously introduced $\mathrm{C} / \mathrm{I}$ and the original proposal based an iterative search of the local maximum for the revenue, from a heuristic starting point in which the marginal utilities are allocated to the value $\vartheta=0.5$. With the Acceptance-probability model it is possible to evaluate the earned revenue. This is done in Figure 1 for the flat pricing policy and in Figure 2 for the usage-based policy. In both cases, two different situations have been represented, with 150 and 200 users respectively. 

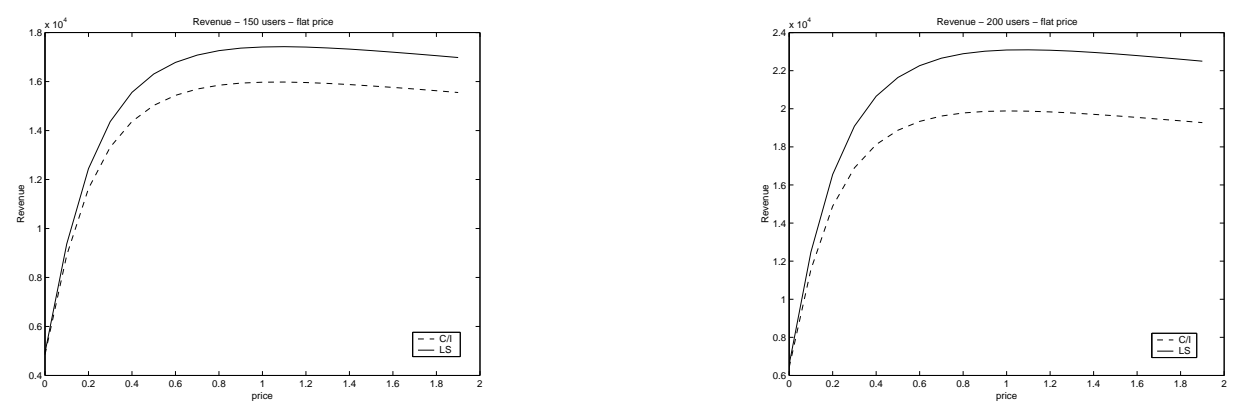

Fig. 1. Revenue for flat price, 150 (left) and 200 (right) users, as a function of the price
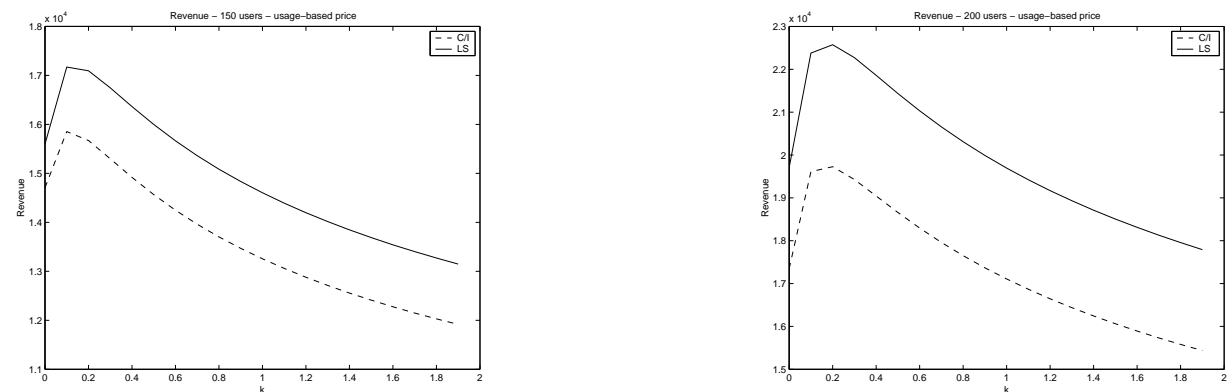

Fig. 2. Revenue for $p(g)=k g, 150$ (left) and 200 (right) users, as a function of $k$

As it was to be expected, the revenue obtained with the local-search strategy (indicated in the Figures as "LS") is better than for the C/I strategy. In fact, the former searches for solutions that improve the revenue. However, note that the qualitative behaviour is similar. Moreover, simulations also show that the number of iterations of the local-search procedure is low (usually 4-5 iterations); thus, it might be said that a simple variation from the initial solution allows to greatly improve the earned revenue ${ }^{1}$. With the LS strategy we are able to obtain a revenue improvement of $10 \%$ approximately in the case with 150 users for both pricing policies. For the 200 users' network, the improvement is even larger, being greater than the $20 \%$. In this way the provider welfare is increased, by leading to a more efficient resource usage, at least from the operator's point-of-view.

As a further observation, note that the curves present an optimal price in both strategies. The existence of a price value that maximises the revenue comes directly from the conditions given by Equation (6). However, the fact that the maximising price is approximately the same for both curves implies that the gap is not due to a different behaviour with respect to the price, but it is structural. In other words, for the same pricing conditions, the LS strategy is able to achieve a higher revenue since it allocates the resources in a more satisfactory way for the users.

In Figure 3, the admission rate is represented, i.e., the percentage of users which are satisfied and achieve a rate $g$ larger than 0 . The acceptance probability model is used here to determine whether a user is satisfied or not. This satisfaction rate can be equivalently seen as admission rate, since users with very low assignments are likely to

\footnotetext{
${ }^{1}$ Note that the most interesting price region is the one which leads to the highest values of the revenue. Here, the improvement is obviously more consistent.
} 


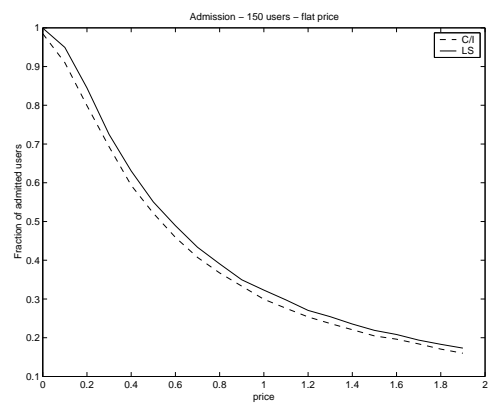

Fig. 3. Admission rate for flat price, 150 users, as a function of the price
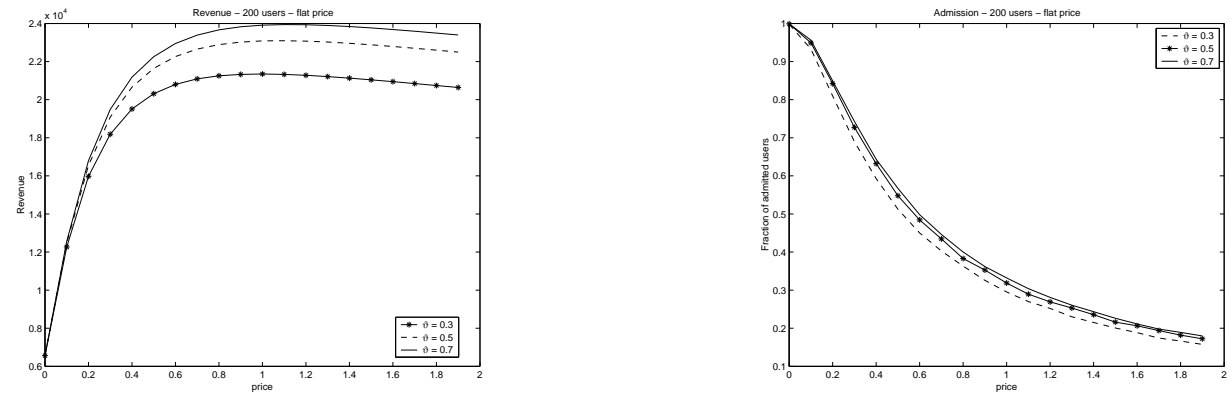

Fig. 4. Revenue (left) and Admission rate (right) for 200 users and variable $\vartheta$

be unsatisfied, so they can be considered as not admitted. In the most extreme case, a user with assigned rate equal to 0 can be seen as a user surely blocked. Here, 150 users and flat price policy are depicted; however, the curves are similar also for different number of users or pricing strategy. It can be observed that the larger the price, the lower the admission rate. Yet, it should also be noted that the LS strategy allows a larger admission rate than the $\mathrm{C} / \mathrm{I}$ strategy. This is due to the revenue improvement that the LS strategy tries to accomplish. In other words, not only the revenue, but also the total users' satisfaction is larger with the LS strategy. This confirms that the LS strategy succeeds in achieving a higher revenue for the operator without hurting the users.

Finally, in Figure 4 the sensitivity of the results to the value of $\vartheta$ is highlighted. Here, three cases $(\vartheta=0.3,0.5,0.7)$ are shown for both revenue and admission rate in a flat price situation with 200 users. The situation with different pricing policies or users' number is yet quite similar. It can be observed that different LS solutions are obtained for different values of the marginal utility starting value $\vartheta$. Even though the qualitative behaviour is similar for the three curves, it can be concluded that a parameter optimisation can improve even further the performance. Thus, a deeper investigation on the effect of the choice of $\vartheta$, in which also considerations about the market strategy of the provider play a role (see [7]) is necessary and can be the subject of a future analysis.

\section{Conclusions and Future Work}

The analysis from the provider's point-of-view of the HSDPA scheduler shows that there are several possibilities of improving the network management under this aspect. This can be easily seen with the introduction of the Acceptance-probability model, 
which considers the joint effect of user utility and price, by allowing to account for economic considerations.

The results show that the application of a classical efficient strategy, like the C/I scheduler, by neglecting the economic counterpart of the allocation, can lead to unsatisfactory results for the operator, even though the C/I strategy provides a maximised throughput. On the other hand, a simple strategy that locally searches for higher values of the revenue is able to greatly improve the profit and the economic efficiency of the resource management, by keeping the users' satisfaction level almost constant, if not increased. Thus, the usefulness of the economic considerations is emphasised. Besides, several further observations open up on how the network welfare can be improved. For example, the simple heuristic strategies discussed here offer the advantages of simplicity and fast evaluation; however, the optimisation of the internal parameters can improve even further the performance and/or the convergence rate.

Finally, from a theoretical point-of-view it could be possible to study within the given framework the behaviour of a more general scheduling strategy, in which the revenue maximisation is considered as the goal of the optimisation problem (8)-(9). This study, that can allow to gain a better understanding of the RRM issues, is left for future research.

\section{References}

1. C. Fragouli, V. Sivaraman, M. Srivastava, "Controlling Multimedia wireless link via enhanced class-based queueing with channel state dependent packet scheduling," Proceedings of INFOCOM 1998, Joint conference of IEEE Computer and Communication Society, Volume 2, 1998.

2. M. Xiao, N.B. Shroff, E.K.-P. Chong, "Utility-Based Power Control in Cellular Wireless Systems," Proceedings of INFOCOM 2001, Joint Conference of the IEEE Computer and Communication Societies, pp 412-421, 2001.

3. L. Song, N. Mandayam, "Hierarchical SIR and Rate Control on the Forward Link for CDMA Data Users under Delay and Error Constraints," IEEE JSAC, IEEE Journal on Selected Areas in Communications, Volume 19, Issue 10, pp 1871-1882, 2001.

4. A. Demers, S. Keshav, S. Shenker, "Analysis and simulation of a fair queuing algorithm," Proceedings of ACM SIGCOMM 1998, Conference on Applications, Technologies, Architectures and Protocols for Computer Communication, pp 1-12, 1998.

5. J.C.R. Benner, H. Zhang, " $\mathrm{WF}^{2} \mathrm{Q}$ : worst case fair weighted fair queuing," Proceedings of INFOCOM 1996, Joint Conference of the IEEE Computer and Communication Societies, pp 120-128, 1996.

6. F.P. Kelly, A. Maulloo, D. Tan, "Rate control in communication networks: shadow prices, proportional fairness and stability", Journal of the Operational Research Society, Volume 49, pp 237-252, 1998.

7. L. Badia, M. Lindström, J. Zander, M. Zorzi, "Demand and Pricing Effects on the Radio Resource Allocation of Multimedia Communication Systems," Proceedings of GLOBECOM 2003 (to appear), 2003.

8. H.R. Varian, "Intermediate Microeconomics: A Modern Approach," Norton, New York, 1999.

9. D. Famolari, N. Mandayam, D. Goodman, V. Shah, "A New Framework for Power Control in Wireless Data Networks: Games, Utility and Pricing”, Wireless Multimedia Network Technologies, Kluwer Academic Publishers, pp 289-310, 1999.

10. E.W. Fulp, M. Ott, D. Reininger, D.S. Reeves, "Paying for QoS: an optimal distributed algorithm for pricing network resources," Proceedings of IWQoS98, International Workshop on Quality of Service, pp 75-84, 1998.

11. T.J. Moulsley, "Performance of UMTS HSDPA for Data Streaming," Proceeding of Third International Conference on 3G Mobile Communication Technologies, 2002.

12. C. Courcoubetis, F.P. Kelly, V.A. Siris, R. Weber, "A study of simple usage-based charging schemes for broadband networks," Telecommunications Systems, Volume 15, pp 323-343, 2000 . 